\title{
Acoustic Casimir pressure for arbitrary media
}

\author{
J. Bárcenas, L. Reyes, and R. Esquivel-Sirvent ${ }^{\text {a) }}$ \\ Instituto de Física, Universidad Nacional Autónoma de México, Apartado Postal 20-364, \\ Ciudad Universitaria, México, 01000, México
}

(Received 12 March 2004; revised 8 May 2004; accepted 11 May 2004)

\begin{abstract}
In this paper we derive a general expression for the acoustic Casimir pressure between two parallel slabs made of arbitrary materials and whose acoustic reflection coefficients are not equal. The formalism is based on the calculation of the local density of modes using a Green's function approach. The results for the Casimir acoustic pressure are generalized to a sphere/plate configuration using the proximity theorem. (C) 2004 Acoustical Society of America.
\end{abstract}

[DOI: 10.1121/1.1768253]

PACS numbers: 43.25.Qp [RR]

Pages: 717-720

\section{INTRODUCTION}

Due to quantum vacuum fluctuations, two parallel neutral plates will attract each other. This phenomenon is known as the Casimir force. ${ }^{1}$ Although a small force, it has been measured accurately using torsion balances, atomic force microscopes, and micromechanical oscillators. ${ }^{2-5}$ An acoustic analog to the Casimir effect was reported in 1996 by Larraza and collaborators, ${ }^{6-8}$ where two parallel plates, placed at a distance $L$, were subjected to a broadband noise background. The plates were observed both to attract and to repel each other, depending on the separation distance and the broadband noise cutoff frequencies. Following Casimir's method, ${ }^{1}$ a theory for the acoustic Casimir force was developed assuming perfectly reflective plates with approximations that turned out to be valid for the frequency range, material, and plate thickness that were used in the experiment. In this work we derive a general expression for the acoustic Casimir pressure for materials with arbitrary impedances by calculating the density of modes between the plates using the Green's function formalism borrowed from the electromagnetic case. We also present an acoustic analog to the proximity theorem to calculate the Casimir pressure between a sphere and a plate.

\section{THEORY}

Consider two different parallel slabs labeled $i=1,2$ of thickness $d_{1,2}$, separated by a distance $L$ along the $z$ axis. The slabs are parallel to the $x-y$ plane and have an arbitrary acoustic reflectivity $r_{1,2}$ [Fig. 1(a)].

For a perfect acoustic reflector, the radiation pressure of a wave of intensity $I$ and speed $c$ impinging on the slab is given by ${ }^{6,9}$

$$
P=\frac{2 I}{c} \cos ^{2}(\theta),
$$

where $\theta$ is the angle of incidence.

${ }^{a)}$ Electronic mail: raul@fisica.unam.mx
As in Refs. 6-8 we consider broadband acoustic noise of constant spectral intensity $I_{\omega}$ in the frequency interval $\left[\omega_{1}, \omega_{2}\right]$, and its spectral representation in the wave vector space

$$
I_{k}=\frac{c I_{\omega}}{4 \pi k^{2}}
$$

where $k^{2}=(\omega / c)^{2}=k_{x}^{2}+k_{y}^{2}+k_{z}^{2}$.

The total radiation pressure on a plate for perfect reflectors, using Eq. (1), is

$$
P_{0}=\frac{I_{\omega}}{2 \pi^{2}} \int d k_{x} d k_{y} d k_{z} \frac{k_{z}^{2}}{k^{4}} .
$$

Between the plates, the total radiation pressure $P_{\text {in }}$ is determined by the allowed modes that satisfy the boundary conditions at the plate surfaces. If the plates are large enough, $k_{x}$ and $k_{y}$ take on continuous values. For perfect reflectors the normal component of the wave vector takes the values $k_{z}=n \pi / L$, where $n$ is an integer. Thus, the calculation of the energy density is reduced to integration over $k_{x}$ and $k_{y}$ and summation over $n$. The Casimir pressure results from the difference $P_{\text {in }}-P_{0}$.

For arbitrary materials the mode summation is no longer direct, since we are no longer allowed to specify Dirichlet boundary conditions that restrict the allowed modes, and it becomes necessary to calculate the total density of modes $\mathcal{D}\left(k_{z}\right)$ and integrate over all wave vector space. To do this we use a Green's function approach.

The wave equation for the velocity potential can be written as the eigenvalue equation $-\partial_{z}^{2} \phi=k_{z}^{2} \phi$, with eigenvalues $k_{z}^{2}$. Let $\lambda_{n}$ be an eigenvalue for the eigenfunction $\phi_{n}$. In terms of the velocity potential $\phi$, the particle velocity $v_{z}$, the fluid density $\rho$ and with the definitions $v_{z}=\partial_{z} \phi_{n}$ and $p=$ $-\rho \partial_{t} \phi_{n}$ we can write the normal component of the wave stress tensor ${ }^{10}$ as

$$
w_{n}=\frac{\rho}{2}\left(\left(\partial_{z} \phi_{n}\right)^{2}+k_{z}^{2} \phi_{n}^{2}\right)
$$

The total contribution to the stress tensor is obtained by adding up all modes and integrating over all possible values of $k_{z}^{2}$. 

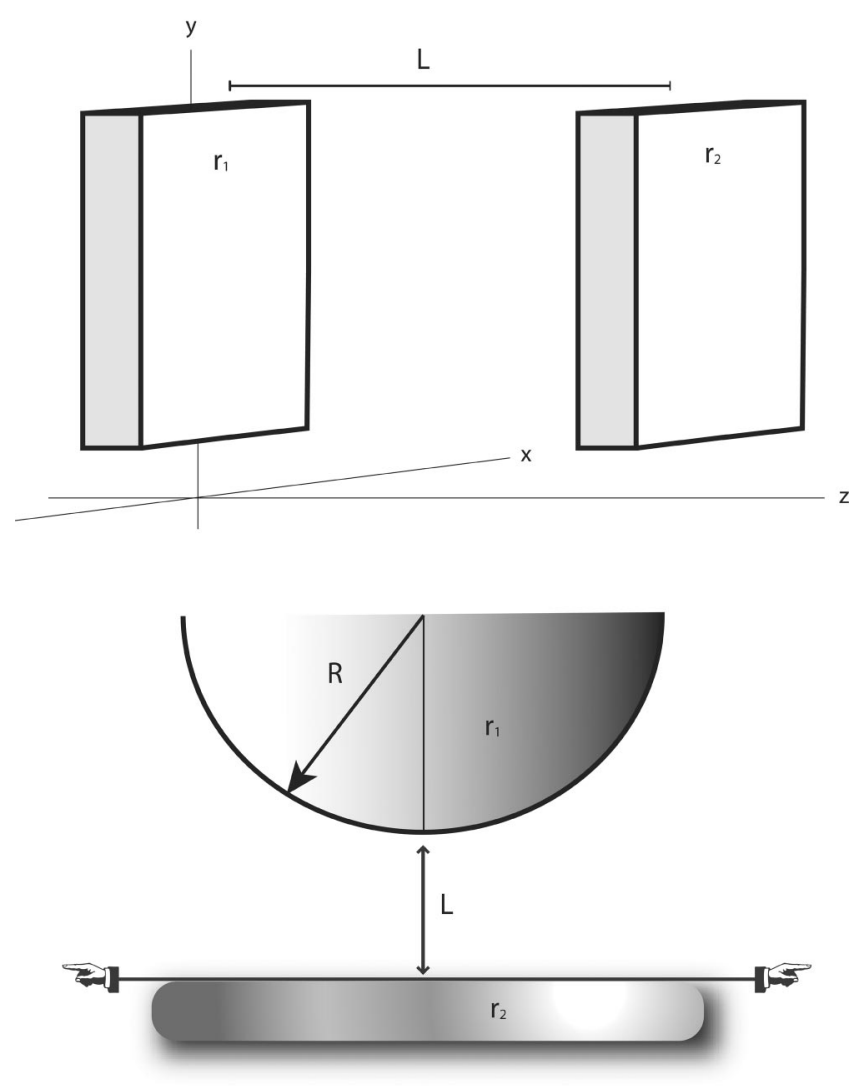

FIG. 1. (a) Geometry and coordinate system for the two parallel plate configuration and (b) for the sphere-plane configuration.

$$
w=\frac{\rho}{2} \int d k_{z}^{2} \sum_{n} \delta\left(k_{z}^{2}-\lambda_{n}\right)\left(\left(\partial_{z} \phi_{n}\right)^{2}+k_{z}^{2} \phi_{n}^{2}\right),
$$

where we have assumed an harmonic behavior of the potential $\phi$ and the Dirac's delta function is introduced since only the eigenmodes contribute to $w$. Now, using the identity

$$
\frac{1}{k_{z}^{+2}-\lambda_{n}}=P \frac{1}{k_{z}^{2}-\lambda_{n}}-i \pi \delta\left(k_{z}^{2}-\lambda_{n}\right),
$$

with $k_{z}^{+2}=\lim _{\eta \rightarrow 0}\left(k^{2}+i \eta\right)$ we can write Eq. (5) as

$$
w=\frac{\rho}{2} \int d k_{z}^{2}\left(-\frac{1}{\pi}\right) \operatorname{Im} \sum_{n} \frac{1}{k_{z}^{+2}-\lambda_{n}}\left(\left(\partial_{z} \phi_{n}\right)^{2}+k_{z}^{2} \phi_{n}^{2}\right) \text {. }
$$

In this equation, we can identify the spectral representation (or eigenfunction expansion) of the Green's function and its derivative, ${ }^{11}$ and we interpret the quantity

$$
\mathcal{D}_{k_{z}^{2}}=-\frac{1}{\pi} \operatorname{Im}\left(G(z, z)+\partial_{z} \partial_{z} G(z, z)\right),
$$

as the density of modes. Another way of understanding the result is as follows. The basic definition of denisty of modes in terms of the Green's funtion is obtained from Eq. (6) as $-\operatorname{Im} G(z, z) / \pi$. The acoustic pressure obeys the wave equation and with the appropriate boundary conditions, we can obtain the Green's function $G_{p}$ and thus the density of modes. From the acoustic stress tensor component [Eq. (4)], besides the pressure field, there is a contribution from the velocity field. Let this field have an associated Green's func- tion $G_{v}$. The total density of modes of the system is $-\operatorname{Im}\left(G_{p}+G_{v}\right) / \pi$. Writing the pressure and velocity in terms of the scalar potential yields Eq. (8). This is equivalent to what happens in zero point Casimir effect where the density of modes comes from adding the contribution of the electric field plus that due to the magnetic fields, and both fields are related through a constitutive equation (Maxwell's equations).

To construct the Green's function for the velocity potential we can use the standard definition

$$
G_{k^{2}}\left(z, z^{\prime}\right)=\frac{\phi^{<}\left(z_{<}\right) \phi^{>}\left(z_{>}\right)}{W},
$$

where $W$ is the Wronskian and

$$
\begin{aligned}
& \phi^{<}(z)=e^{-i k_{z} z}+r_{1} e^{i k_{z} z}, \\
& \phi^{>}(z)=e^{i k_{z}(z-L)}+r_{2} e^{-i k_{z}(z-L)},
\end{aligned}
$$

are the solutions to the one-dimenional wave equation where the superscript $(<,>)$ represents the smaller and larger of $z$ and $z^{\prime}$, respectively.

Substitution of the potentials [Eq. (10)] into Eqs. (9) and (8) yields the local density of modes

$$
\mathcal{D}_{k_{z}^{2}}=\frac{1}{2 k_{z} \pi} \operatorname{Re}\left[\frac{1+r_{1} r_{2} e^{2 i k_{z} L}}{1-r_{1} r_{2} e^{2 i k_{z} L}}\right],
$$

where we have obviated the dependence of the reflectivities with wave vector. The density of states Eq. (11) was obtained from the Green's function for the Helmholtz equation with eigenvalues $k_{z}^{2}$. However, we are interested in the density of states for $k_{z}$. This is simply obtained from $\mathcal{D}_{k_{z}^{2}}=d\left(k_{z}^{2}\right) \mathcal{D}_{k_{z}}$ $=2 k_{z} \mathcal{D}_{k_{z}^{2}}$, or

$$
\mathcal{D}_{k_{z}}=\frac{1}{\pi} \operatorname{Re}\left[\frac{1+r_{1} r_{2} e^{2 i k_{z} L}}{1-r_{1} r_{2} e^{2 i k_{z} L}}\right] \text {. }
$$

The radiation pressure due to the inside modes can now be written as

$$
P_{\text {in }}=\frac{I_{\omega}}{4 \pi} \int d k_{x} d k_{y} d k_{z} \mathcal{D}_{k_{z}} \frac{k_{z}^{2}}{k^{4}}
$$

In the limit of perfect reflectors $r \rightarrow 1$ the density of states becomes $\left(k_{0} / \pi\right) \delta\left(k_{z}-n k_{0}\right)$ where $k_{0}=\pi / L$. Thus, from Eq. (13) the pressure due to all the modes is

$$
P_{\text {in }}=\frac{k_{0} I_{\omega}}{2 \pi^{2}} \int d k_{x} d k_{y} d k_{z} \sum_{n} \delta\left(k_{z}-n k_{0}\right) \frac{k_{z}^{2}}{k^{4}},
$$

or

$$
P_{\text {in }}=\frac{k_{0}^{3} I_{\omega}}{2 \pi} \int d k_{x} d k_{y} \sum_{n} \frac{n^{2}}{\left(k_{x}^{2}+k_{y}^{2}+n^{2} k_{0}^{2}\right)^{2}},
$$

which is the same as that obtained by Larraza and collaborators. ${ }^{6-8}$ Finally, the acoustic Casimir force per unit area $f=P_{\text {in }}-P_{\text {out }}$ takes the form

$$
f=\frac{I_{\omega}}{2 \pi^{2}} \operatorname{Re}\left(\int d k_{x} d k_{y} d k_{z} \frac{k_{z}^{2}}{k^{4}}\left(\frac{1}{\xi-1}\right)\right),
$$




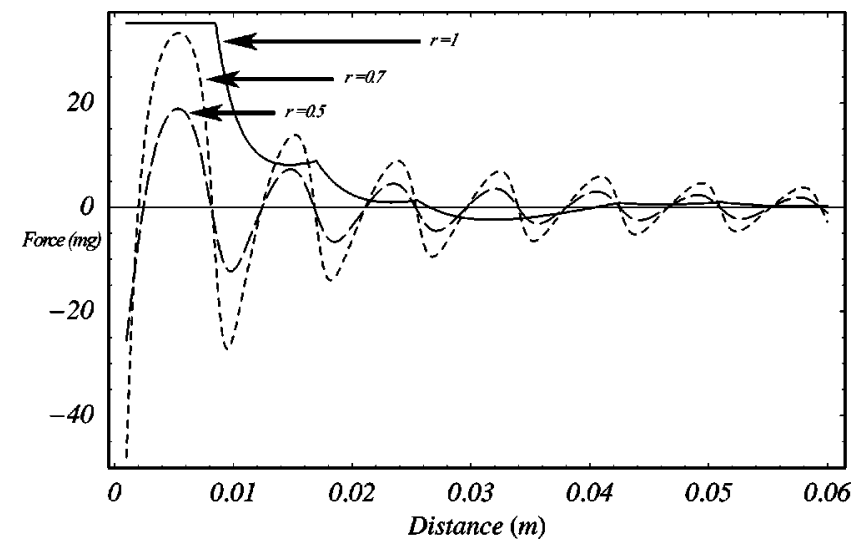

FIG. 2. Acoustic Casimir force between two parallel plates for different values of the reflectivity $r$ assuming both plates are equal. The values of $r$ are indicated. The intensity and bandwidth are the same as in the experiments of Laraza (Ref. 6).

where $\xi=\left(r_{1} r_{2} \exp \left(2 i k_{z} L\right)\right)^{-1}$. Notice that it is enough to know the separation between the slabs and the reflectivities to determine the acoustic Casimir force.

To illustrate the application of Eq. (16), in Fig. 2 we plot the force versus separation for two identical slabs with constant reflectivities $r=1,0.8,0.7$. In all cases the force goes from attractive to repulsive as the separation increases. The magnitude of the force is not only related to the reflectivity but also to the finite bandwidth being used. If the bandwidth extends from zero to infinity, the acoustic Casimir pressure for a perfect reflector $-\pi I_{\omega} / 4 L$ is always attractive. If we integrate Eq. (16) over all frequencies the force is also always attractive and as the reflectivity decreases the force does too ${ }^{12}$ in all cases. Without loss of generality we have assumed a constant value of $r$ within the bandwidth under consideration. However, the formalism is valid even when the reflectivity shows a strong dependence with frequency. The bandwidth and intensity used in these calculations are the same as in Laraza. ${ }^{6}$ Even if we consider a finite frequency bandwidth it is possible to obtain a purely attractive force if we consider the force between a surface with reflectivity $r_{1}=1$ and a pressure release surface $r_{2}$ $=-1$. In this limit the force is constant and always attractive since $D_{k_{z}} \rightarrow 0$, as can be seen from Eq. (12) and the external pressure field pushes the plates together.

\section{THE PROXIMITY THEOREM IN ACOUSTICS}

Practical measurements of the Casimir force due to zero point energy fluctuations are done between a large sphere and a plane due to the difficulty of keeping two plates parallel at the submicron scale. ${ }^{2-5}$ The force between a large sphere and a plane [see Fig. 1(b)] is calculated using the proximity theorem ${ }^{13}$ or Derjaguin approximation, ${ }^{14}$ which states that

the force between two smooth surfaces as a function of the separation degree of freedom is proportional to the interaction potential per unit area $\mathcal{E}$ between two flat surfaces, the proportionality factor being $2 \pi$ times the reciprocal of the square root of the Gasussian cuadra-

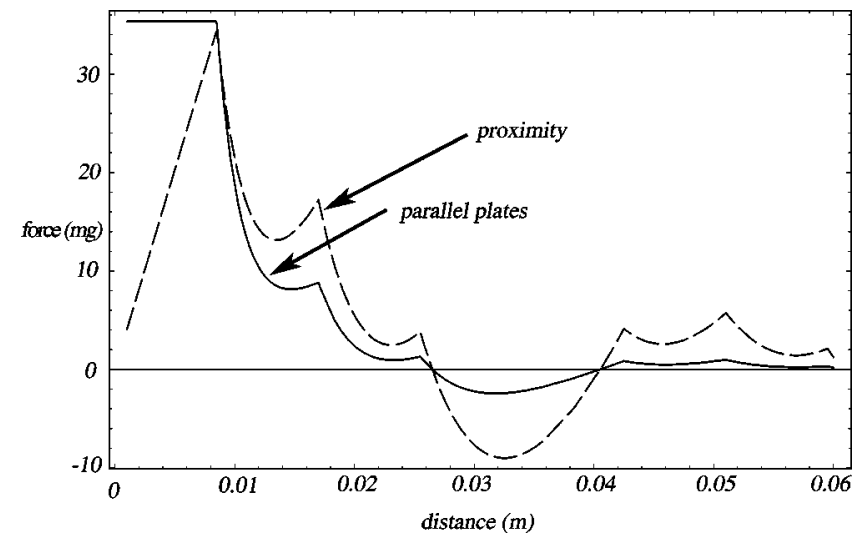

FIG. 3. Acoustic Casimir force between a sphere $(R=0.2 \mathrm{~m})$ and a plane for reflectivity $r=1$ (dashed line). For comparison the force between two parallel plates is also shown (solid line).

ture of the gap width function at the point of closest approach.

For a sphere-plane system, the force $F_{s p}$ is obtained from the Casimir free energy per unit area between two parallel plates $\mathcal{E}$ as

$$
f_{s p}=2 \pi R \mathcal{E},
$$

where $R$ is the radius of the sphere. The proximity theorem is valid provided $L / R<1, L$ being the closest distance between the surface, although the limit of $L \rightarrow 0$ can at be described by the proximity theorem. A current problem $^{15}$ is that there are no bounds on how big $L / R$ has to be in order to obtain the correct result. Experimentally this becomes difficult at the submicron scale. The acoustic analog of the Casimir force provides a simpler (not necessarily easier) way of solving this problem, since as shown by Larraza ${ }^{6}$ the scale of the acoustic experiments allows a more precise control of the involved parameters. Furthermore, the proximity theorem is valid for any interaction. In the acoustic case, the free energy per unit area for parallel plates is

$$
\left.\mathcal{E}=\frac{I_{\omega}}{4 \pi^{2}} \int d k_{x} d k_{y} d k_{z} \frac{k_{z}}{k^{4}} \operatorname{Re}\left(\ln \left(1-r_{1} r_{2} e^{2 i k_{z} L}\right)\right)\right) .
$$

This expression for $\mathcal{E}$ is such that the force [Eq. (16)] is given by $f=-\partial \mathcal{E} / \partial L$. Thus, the force between a sphere and a plane is

$$
\left.f_{p s}=\frac{R I_{\omega}}{2 \pi} \int d k_{x} d k_{y} d k_{z} \frac{k_{z}}{k^{4}} \operatorname{Re}\left(\ln \left(1-r_{1} r_{2} e^{2 i k_{z} L}\right)\right)\right) .
$$

To show the application of this approximation, in Fig. 3 we have plotted the force between two parallel plates and the force between a $20 \mathrm{~cm}$ sphere and a plate. We observe that although the proximity theorem gives a correct behavior and overall order of magnitude for the force, the region in the limit of $L$ approaching zero is not well described.

\section{CONCLUSIONS}

We have derived a general expression for the acoustic Casimir force between two parallel slabs with arbitrary acoustic properties characterized by the reflection coefficients of the material. We also extended our results to include 
the force between a sphere and a plane. The expression we obtain for the Casimir force is convenient for calculations since it depends mainly on the reflection coefficients that can be obtained straightforwardly in theoretical computations or through experimental work. Our approach is analogous to the electromagnetic dielectric case, so this formalism is equivalent to the Liftshitz formula. ${ }^{16,17}$ In the limit of a perfectly reflective plate our results agree with those of Larraza. ${ }^{6}$ This formalism can be extended to the case of highly porous materials or viscous propagation media, although the calculations involved can be of increasing difficulty. It must be pointed out that for the case when the material is deformed by the wave, this density of states approach is no longer valid: the reflection coefficient is angular dependent, and since the angle itself is time dependent the use of a static density of states would be incorrect. Also, we have excluded the possible effects of roughness.

The crucial difference between the system we consider and the original treatment ${ }^{6}$ is the inclusion of the density of states through the Green's function method. The analytical interpretation of the density function gives a deeper insight into what really happens in a nonperfect reflector. For a perfect reflector the density of modes consists of a series of Dirac's deltas. As the reflectivity decreases from unity, the resonance bands increase in width, which is heuristically equivalent to a spatial diffusion of the nodes that appear inside the resonant cavities.

Although the use of perfectly reflectiving plates is a good approximation in some experimental situations, this is not the case for other bodies (such as rubber, as an extreme example) hence our efforts to broaden the horizon of application. As an example, we have considered the possibility of using acoustic experiments to prove the validity of the proximity theorem. Additionally, this treatment could allow for a larger range of experimental versus theoretical comparison in this field where, as noted by Larraza et al., the possibility of direct technological application of the acoustic Casimir effect is considerable.

\section{ACKNOWLEDGMENT}

Partial support provided by DGAPA-UNAM Project No. IN116002-2.

${ }^{1}$ H. B. G. Casimir, "On the attraction between two perfectly conducting plates," Proc. K. Ned. Akad. Wet. 51, 793 (1948).

${ }^{2}$ S. K. Lamoreaux, "Demonstration of the Casimir force in the 0.6 to $6 \mu \mathrm{m}$ range," Phys. Rev. Lett. 78, 5 (1997).

${ }^{3} \mathrm{U}$. Mohideen and A. Roy, "Precision measurement of the Casimir force from 0.1 to $0.9 \mu \mathrm{m}$," Phys. Rev. Lett. 81, 4549 (1998).

${ }^{4}$ B. W. Harris, F. Chen, and U. Mohideen, "Precision measurement of the Casimir force using gold surfaces," Phys. Rev. A 62, 052109 (2000).

${ }^{5}$ H. B. Chan, V. A. Aksyuk, R. N. Kliman, D. J. Bishop, and F. Capasso, "Quantum mechanical actuation of microelectromechanical systems by the Casimir force," Science 291, 1942 (2001).

${ }^{6}$ A. Larraza, C. D. Holmes, R. T. Susbilla, and B. Denardo, "The force between two parallel rigid plates due to the radiation pressure of broadband noise: An acoustic Casimir effect," J. Acoust. Soc. Am. 103, 2267 (1998).

${ }^{7}$ A. Larraza and B. Denardo, "An acoustic Casimir effect," Phys. Lett. A 248, 151 (1998).

${ }^{8}$ A. Larraza, "A demonstration apparatus for an acoustic analog to the Casimir effect," Am. J. Phys. 67, 1028 (1999).

${ }^{9}$ L. D. Landau and E. M. Lifshitz, Fluid Mechanics (Pergamon, New York, 1987).

${ }^{10}$ C. P. Lee and T. G. Wang, “Acoustic radiation pressure," J. Acoust. Soc. Am. 94, 1099-1109 (1993).

${ }^{11}$ A. Gonis, Theoretical Materials Science (Materials Research Society, Warrendale, PA 2000).

${ }^{12}$ When the bandwidth extends from zero to infinity it is possible to evaluate the force by mapping the real frequency axis $\omega$ to $i \omega$, making the integral numerically stable. See, for example, Ref. 17.

${ }^{13}$ L. R. White, "On the Deryaguin approximation for the interaction of macrobodies," J. Colloid Interface Sci. 95, 286 (1983).

${ }^{14}$ J. Blocki, J. Randrup, W. J. Swiatecki, and C. F. Tsang, "Proximity forces," Ann. Phys. (N.Y.) 105, 427 (1977).

${ }^{15}$ C. Roman-Velazquez, C. Noguez, C. Villarreal, and R. Esquivel-Sirvent, "Spectral representation of the nonretarded dispersive force between a sphere and a substrate," Phys. Rev. A (to be published).

${ }^{16}$ W. L. Mochán, C. Villarreal, and R. Esquivel-Sirvent, "On Casimir forces for media with arbitrary dielectric properties," Rev. Mex. Fis. 48, 339 (2002).

${ }^{17}$ R. Esquivel-Sirvent, C. Villarreal, and W. M. Mochan, "Exact surface impedance formulation of the Casimir force: Application to spatially dispersive metals," Phys. Rev. A 68, 052103 (2000). 